\title{
Review of: "The impact of renewable energy and sector coupling on the pathway towards a sustainable energy system in Chile"
}

\section{Muntasir Murshed ${ }^{1}$}

1 North South University

Potential competing interests: The author(s) declared that no potential competing interests exist.

The article is a well-articulated one in respect of attaining energy and environmental sustainability by promoting renewable energy uses in Chile. 